\title{
Enhancement of Crop Images Using Image Fusion Method in Transform Domain
}

\author{
${ }^{* 1}$ Dr. Vijaya Shandilya, ${ }^{2}$ Dr. Shyamkant S.Munje \\ ${ }^{* 1}$ Department of CSE. SIPNA COET, Amravati, India \\ ${ }^{2}$ Department of Entamology, Dr.P.D.K.V, Akola, India \\ Email:vkshandilya@rediffmail.com, shyammunje@yahoo.com
}

\author{
Received: $20^{\text {th }}$ September 2018, Accepted: $11^{\text {th }}$ October 2018, Published: $31^{\text {st }}$ October 2018
}

\begin{abstract}
Computer vision and/or Image processing is the vast field and very much applicative in almost all sectors. We require reliable and accurate data from the images. The information which is extracted from the processed images is used in various domains. Here we chose agriculture domain where images are captured of different crops and then processed those images using image processing algorithm to improve the visual quality of the crop images. Once the clear image is available researcher from agriculture industry can identify problems associated with the crops. We are applying fusion technique to the set of crop images using complex wavelet transform (CWT) from the family of Discrete Wavelet Transform (DWT) implemented using MATLAB. This method enhanced the overall outcome of the fused image in terms of spectral and spatial characteristics of the image.
\end{abstract}

\section{Keywords}

Image Fusion, CWT, DWT, MATLAB.

\section{Introduction}

The field of image processing has the wide range of applications where the outcome of processing in some desired form is given to the end user of the system. Image fusion is one of the method of image processing only the case is it involves multiple images whereas other processing mostly wok on single image. Fusion use images from single sensors to multiple sensors. It fuses the number of images using some fusion methodology to come up with accurate and reliable results [1][2]. Sometime information from single sensor is not clearly visible or absent, we can use images from different sensors to perceive this information.

There are various application area were image fusion is prominently working such as [3], Military and law enforcement, Medical imaging, Robotic, Agriculture - crop analysis, crop disease identification, Remote sensing and so on.

The proposed method work for most of the applications; where the images are multi-focus images, multi-view images and multi-modal images. Fusion is performed when the input images are not clear and user/ analyst are not able to provide information from it. It is very much helpful in the blurred images as we have number of images of same scene we can obtain its clear view by fusing partial blurred images or any combinations. Here we have chosen its most demanding application i.e. agriculture because it has direct benefit to the farmers and the society. Image Fusion is the process which provides clear and informative view of the field or crop under observation from number of images which are submitted as an input. Wide ranges of sensors are available nowadays which will provide crop images in different wavelengths. We can use those sensors to collect images and perform some processing and finally extract useful data from it. This data ultimately assist to agricultural scientist to identify certain problem or situation and finally serve the farmer and society. Manual analysis of these images is the cumbersome job. So we are applying fusion algorithm. This paper elaborates the method for fusion of number of images into one composite image which is accurate, reliable and full of information. The algorithm is based on complex wavelet transform (CWT) from the family of Discrete Wavelet Transform (DWT) implemented using MATLAB. This method enhanced the overall outcome of the fusion in terms of spectral and spatial characteristics of the image.

Image which is the main input of the overall processing is better defined by its resolution. Resolution means the details an image hold. There are four resolutions in which an image is defined such as spatial resolution, spectral resolution, radiometric resolution and temporal resolution [4]. We can improve these resolutions to get benefits of the image fusion process.

The benefits of image fusion include [5] Reduced uncertainty, Increased reliability, Robust system performance, Compact representation of information, Extended spatial and temporal coverage. 


\section{Methods and Design Issues:}

The paper is mainly focusing on various aspects such as the fused images of crops must be clear and accurate, also they must be reliable so that further analysis of those images can provide correct information about the crop health. Its result can also be utilized in identifying certain diseases on plant. For an image to be accurate and reliable it must preserve both spectral as well as spatial characteristics of the source images. Basically in this

domain of crop analysis it is required that final image should not contain artifacts and noise as it distracts the analyst's perception.

While designing the fusion methodology role of spatial and spectral information of image must be considered to attend the objectives of fusion as accuracy, reliability, informative output image and so on. From the literature survey a promising technique comes out for image fusion i.e. wavelet transform technique. Discrete Wavelet Transform (DWT) preserves more spectral characteristics. But has some fundamental problems which affect the fusion outcome as Lack of shift invariance, Poor directional selectivity [6] [7]. Whereas CWT is the complex-valued extension to the standard discrete wavelet transform. It is shift invariant and directionally selective [6] [7].

The research work uses Complex Wavelet Transform along with the anisotropic multi-directional wavelet transform method for fusion of multiple images.

\section{Proposed Methodology}

The steps of proposed fusion method are given below:

Prerequisite is that the two input images must be registered before fusion process is performed.

Step 1: Read the two images (images must be registered one). Here we can also input Low resolution Multispectral image and High resolution panchromatic image.

Step 2: Pre-processing: In this step wiener filter of kernel is applied to remove noise from the images. Noisy image leads to incorrect assumption during fusion process.

Step 3: Separation of images into RGB component plane: Divide the images into R component plane, G component plane, and $\mathrm{B}$ component plane.

Step 4: Decomposition step: Apply the decomposition method to R, G \& B component images of both the images separately. For decomposition complex wavelet transforms with the properties of direction anisotropic wavelet transform is applied. Here we have assumed 16 directions of decomposition with 4 layers.

Angles are varying in the range of 0 to $(2 * \mathrm{Pi}-\mathrm{Pi} / 8)$ in the step of $\mathrm{Pi} / 8$.

So the range of angles we get is $0,22.5,45,67.5,90,112.5,135,157.5,180,202.5,225,247.5,270,292.5,315$, and 337.5.

Step 5: Fuse the component images: here fuse the R-R component image of I1 and I2, G-G component image of I1 and I2, B-B component images of I1 and I2 separately. To perform this fusion we have calculated matching parameter which will check matching factor of both the sub-bands of images I1 \& I2, and high coefficient edge information of sub-bands. Using these values we have generated a threshold value.

- If the sub-band images are not similar then the threshold is considered as high coefficient edge information of I1 or I2 whichever is larger.

- If the sub-band images are similar then the threshold is considered as mixture of high coefficient edge details of source images. Weights applied to them.

The respective component planes are then fused.

Step 6: The component planes are respectively fused together i.e. R, G, and B of the source images.

Step 7: The step is the inverse reconstruction to obtain the final output fused image

\section{Results and Discussion}

Proposed methodology is tested on various sets of images and performance parameters are evaluated. A sample of image fusion applied on plant images is given in Fig. 1 below. These two images i.e. (a) \& (b) are multi-focus images of plant having some blurred portion which are further fused together to get clear image as shown in (c). We can observe that fused image is having all objects very clear. Similarly the proposed method is also tested on other applications, sample test result images are given in Fig. 2 to Fig. 5. Those images are belonging to Multi-focal or blurred or Multi-modal images group. 


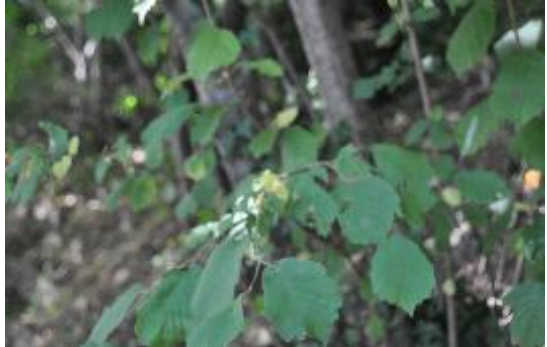

(a)

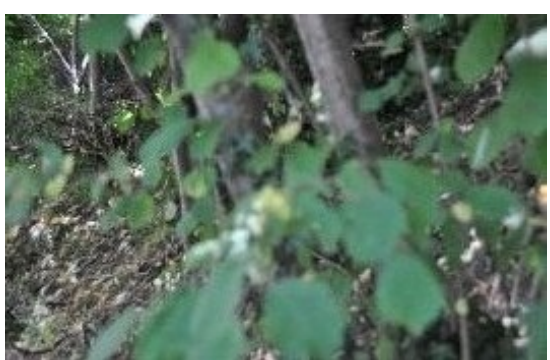

(b)

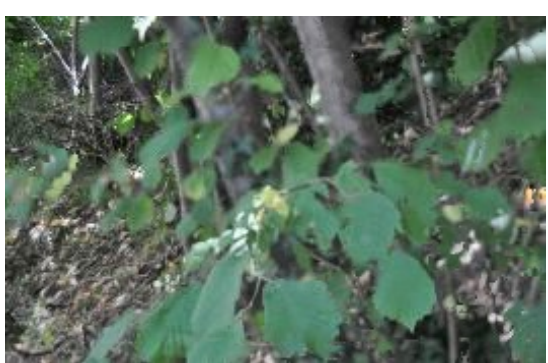

(c)

Figure 1: Data Set 1 of Multi-focus Images (a) Plant Image 1 (b) Plant Image 2 (c) Fused Image Using Proposed Method

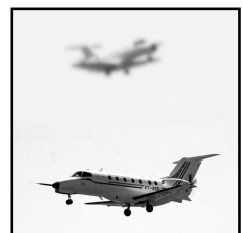

(a)

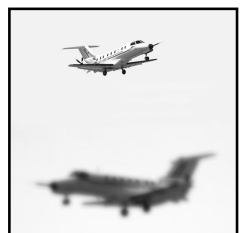

(b)

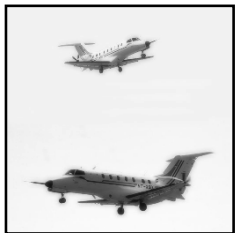

(c)

Figure 2: Data Set 2 of Air Plane Blur Images (a) Source Image I (b) Source Image II (c) Fused Image Using Proposed Method

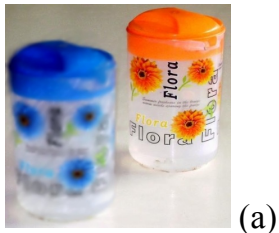

(a)

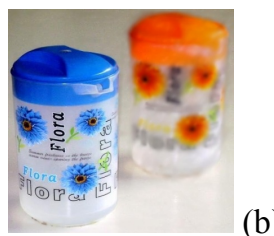

(b)

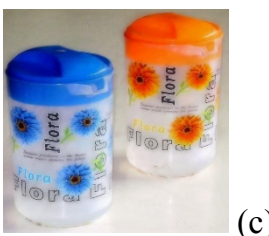

(c)

Figure 3: Data Set 3 of Multi-focus Blur Images (a) Source Image I (b) Source Image II (c) Fused Image Using Proposed Method

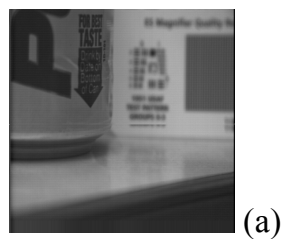

(a)

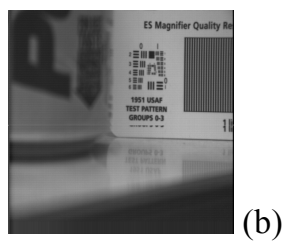

(b)

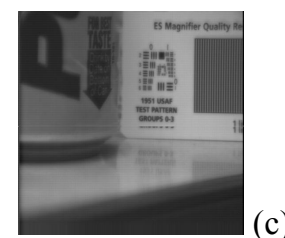

(c)

Figure 4: Data Set 4 of Multi-focus Images (a) Source Image I (b) Source Image II (c) Fused Image Using Proposed Method

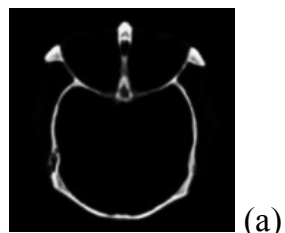

Figure 5: Data Set 5of Multi-Modal Images (a)

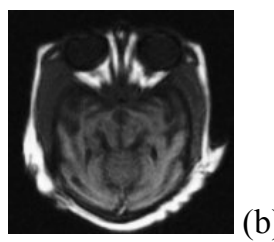

(b)

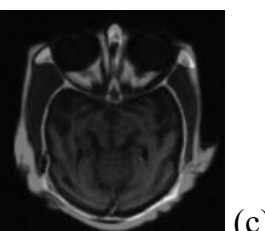

(c)

Method

Results are tested and compare with other methods as PCA and Weighted average method. We have used various wavelets while implementing these methods as HAAR Wavelet, Daubechies Wavelet (db4), Coiflet Wavelet (coif1), Symlets Wavelet (Sym2). We have used PCA method because it is the classical method and its results are good in spatial characteristics of an image. A weighted average method is also spatial domain technique. These methods are very simple, easy to understand and implement. But it reduces the resultant image quality consequently by introducing noise into fused image. It leads to unwanted side effects like reduced contrast. Wavelet based methods preserves more spectral information but looses more spatial information. A more or less level of decomposition leads to degeneration of either spatial 
quantity or spectral characteristics. Complex Wavelet transform method is the good trade between the spectral information of multispectral image and spatial information of the panchromatic band.

Evaluation of fusion method is shown in following figures 6 to 9 which shows the comparative analysis of the evaluation parameters PSNR (Peak Signal to Noise Ratio), SSIM (Structural Similarity Index Measure), CORR (Correlation), MAE (Mean Absolute Error).

Data sets used in this paper are collected from Agriculture University. We have also evaluated this method for images other than agriculture images which are blurred and multi-focus (shown in Tables 1 to 4).

\begin{tabular}{|c|c|c|c|c|c|c|}
\hline FUSION METHOD & WAVELET & Data set 1 & Data set 2 & Data set 3 & Data set 4 & Data set 5 \\
\hline \multirow{4}{*}{ PCA Fusion Method } & Haar & 35.214 & 32.568 & 33.453 & 37.186 & 30.169 \\
\cline { 2 - 7 } & db4 & 35.214 & 33.682 & 33.453 & 37.179 & 30.163 \\
\cline { 2 - 7 } & coif1 & 35.214 & 32.567 & 33.453 & 37.182 & 30.168 \\
\cline { 2 - 7 } & sym2 & 35.214 & 32.567 & 33.454 & 37.184 & 30.17 \\
\hline \multirow{3}{*}{$\begin{array}{c}\text { Weighted Average } \\
\text { Fusion Method }\end{array}$} & Haar & 35.217 & 32.577 & 33.456 & 37.151 & 28.905 \\
\cline { 2 - 7 } & db4 & 35.204 & 32.564 & 33.446 & 37.169 & 28.884 \\
\cline { 2 - 7 } & coif1 & 35.205 & 32.564 & 33.445 & 37.155 & 28.885 \\
\hline & sym2 & 35.204 & 32.564 & 33.446 & 37.154 & 28.886 \\
\hline \multirow{2}{*}{ Proposed Method } & $\begin{array}{c}\text { CWT with } \\
\text { AWT }\end{array}$ & 38.446 & 34.917 & 37.526 & 40.274 & 29.593 \\
\hline
\end{tabular}

Table 1. PSNR Evaluation Parameters for Weighted average, PCA and Proposed method for data set 1 to 5.

\begin{tabular}{|c|c|c|c|c|c|c|}
\hline FUSION METHOD & WAVELET & Data set 1 & Data set 2 & Data set 3 & Data set 4 & Data set 5 \\
\hline \multirow{4}{*}{ PCA Fusion Method } & Haar & 0.8968 & 1 & 0.9973 & 0.7588 & 1 \\
\hline & db4 & 0.8966 & 1 & 0.9973 & 0.7583 & 1 \\
\hline & coif1 & 0.8966 & 1 & 0.9973 & 0.7587 & 1 \\
\hline & sym2 & 0.8967 & 1 & 0.9973 & 0.7586 & 1 \\
\hline \multirow{4}{*}{$\begin{array}{l}\text { Weighted Average } \\
\text { Fusion Method }\end{array}$} & Haar & 0.8959 & 1 & 0.9973 & 0.7521 & 1 \\
\hline & db4 & 0.8958 & 1 & 0.9973 & 0.7564 & 1 \\
\hline & coif1 & 0.8957 & 1 & 0.9973 & 0.7533 & 1 \\
\hline & sym2 & 0.8957 & 1 & 0.9973 & 0.7519 & 1 \\
\hline Proposed Method & CWT with AWT & 0.9691 & 1 & 0.9977 & 0.9491 & 1 \\
\hline
\end{tabular}

Table 2. SSIM Evaluation Parameters for Weighted average, PCA and Proposed method for data set 1 to 5.

\begin{tabular}{|c|c|c|c|c|c|c|}
\hline \multirow{2}{*}{ FUSION METHOD } & WAVELET & Data set 1 & Data set 2 & Data set 3 & Data set 4 & Data set 5 \\
\hline \multirow{4}{*}{ PCA Fusion Method } & Haar & 0.9964 & 0.9933 & 0.9874 & 0.9931 & 0.8904 \\
\cline { 2 - 7 } & db4 & 0.9964 & 0.9959 & 0.9874 & 0.9931 & 0.8896 \\
\cline { 2 - 7 } & coif1 & 0.9964 & 0.9933 & 0.9874 & 0.9931 & 0.8901 \\
\cline { 2 - 7 } & sym2 & 0.9964 & 0.9933 & 0.9874 & 0.9931 & 0.8903 \\
\hline \multirow{3}{*}{$\begin{array}{c}\text { Weighted Average } \\
\text { Fusion Method }\end{array}$} & db4 & 0.9964 & 0.9934 & 0.9874 & 0.993 & 0.7541 \\
\cline { 2 - 7 } & $\mathbf{c o i f 1}$ & 0.9964 & 0.9933 & 0.9873 & 0.9931 & 0.751 \\
\cline { 2 - 7 } & sym2 & 0.9964 & 0.9933 & 0.9873 & 0.993 & 0.7512 \\
\hline \multirow{2}{*}{ Proposed Method } & $\begin{array}{c}\text { CWT with } \\
\text { AWT }\end{array}$ & 0.9992 & 0.9978 & 0.99873 & 0.993 & 0.7513 \\
\hline
\end{tabular}

Table 3. CORR Evaluation Parameters for Weighted average, PCA and Proposed method for data set 1 to 5. 
Helix Vol. 8(6): 4353- 4357

\begin{tabular}{|c|c|c|c|c|c|c|}
\hline \multirow{2}{*}{ FUSION METHOD } & WAVELET & Data set 1 & Data set 2 & Data set 3 & Data set 4 & Data set 5 \\
\hline \multirow{3}{*}{ PCA Fusion Method } & Haar & 6.4848 & 34.9629 & 34.2672 & 6.9781 & 54.9581 \\
\cline { 2 - 7 } & $\mathbf{d b 4}$ & 6.4923 & 26.2361 & 34.2866 & 6.9832 & 55.2381 \\
\cline { 2 - 7 } & $\mathbf{c o i f 1}$ & 6.4923 & 34.9772 & 34.2732 & 6.979 & 55.1211 \\
\cline { 2 - 7 } & $\mathbf{s y m 2}$ & 6.4914 & 34.9764 & 34.2665 & 6.9783 & 55.0886 \\
\hline \multirow{3}{*}{$\begin{array}{c}\text { Heighted Average } \\
\text { Fusion Method }\end{array}$} & $\mathbf{d b 4}$ & 6.4788 & 34.7704 & 34.225 & 7.0314 & 90.529 \\
\cline { 2 - 7 } & $\mathbf{c o i f 1}$ & 6.517 & 34.9681 & 34.3322 & 7.003 & 90.6333 \\
\cline { 2 - 7 } & $\mathbf{s y m 2}$ & 6.5163 & 34.9649 & 34.3235 & 7.0468 & 90.6377 \\
\hline Proposed Method & $\begin{array}{c}\text { CWT with } \\
\text { AWT }\end{array}$ & 2.9809 & 21.945 & 12.3153 & 3.4396 & 84.2691 \\
\hline
\end{tabular}

Table 4. CORR Evaluation Parameters for Weighted average, PCA and Proposed method for data set 1 to 5.

In this paper we have primarily focused on Multi- focus Fusion. Multi-focus are the source images such that every region is in focus in at least one source image or channel. Due to the limited focus depth of the optical lens it is often not possible to get an image that contains all relevant objects in focus. To obtain an image with every object in focus a multi-focus image fusion process is required to fuse the images giving a better view for human or machine perception [8].

The assessment parameters shown above are to understand their contribution in performance of the proposed fusion method.

Reliability of the method is better justified by MAE. PSNR gives the Accuracy of the method and structural Information content is evaluated by SSIM and CORR shows similarity between original images and the final fused image.

While evaluating these it is observed that some results of few parameters are inferior for some dataset specifically for data set 5 it is because data set 5 images belongs to medical domain which has some fine spatial resolution details which is not properly captured in proposed method. Here proposed method is slightly need to modify as per the domain and application. But above all proposed method proves to be the best in capturing rest of the details.

\section{Conclusion}

The proposed method can effectively use the complementary and redundant information of source images. The evaluation parameters demonstrate that the fused image preserve spectral as well as spatial characteristics of the source images. The method use strong edge features to enhance the final fused image. The proposed method also possesses property of perfect reconstruction which yield good fusion result compare to classical fusion method such as PCA [9].

Once the enhanced and clear image is available researcher from agriculture industry can perform various tasks on it. They can analyze crop health, crop density and coverage; stages of plant growth, various diseases associated with the crops and can provide solutions to them.

\section{References}

[1] Yufeng Zheng, Image Fusion and Its Applications, Edited by, Alcorn State University, USA, ISBN 978-953-307182-4, Hard cover, 242 pages, Publisher: InTech, Chapters published June 24, 2011 under CC BY-NC-SA 3.0 license DOI: $10.5772 / 691$.

[2] Guest editorial, Image fusion: Advances in the state of the art, Science Direct Information Fusion Vol.8 (2007) Pg.114-118, ISSN 1556-2535.

[3] Investigations of Image Fusion, Electrical Engineering and Computer science and Department, Lehigh University, Bethlehem, www.ece.lehigh.edu/SPCRL/image_fusion.htm.

[4] Dr. S.C. Liew, Principles of Remote sensing, www.crisp.nus.edu.sg/ resesrch/tutorial/ rsmain.htm, Centre for Remote Imaging, Sensing and Processing (CRISP) 1997, 2001.

[5] H.B. Mitchell, Image Fusion - Theories, Techniques and Applications, ISBN 978-3-642-11215-7, e-ISBN 978-3-64211216-4,DOI 10.1007/978-3-642-11216-4, 2010 Springer

[6] Xu Qing, Xing Shuai, Tan Bing, Li Jiansheng, Geng Zexun, COMPLEX WAVELETS AND ITS APPLICATION TO IMAGE FUSION, International Society for Photogrammetry and Remote Sensing, www.isprs.org/ proceedings/XXXV/ congress/ comm3/ papers/ 326.pdf

[7] N. Kingsbury, The dual-tree complex wavelet transform: a new technique for shift invariance and directional filters. In IEEE Digital Signal Processing Workshop, volume 86, 1998.

[8] AMR NASR, A Comparative Analysis of Image Fusion Methods, www.slideshare.net, Feb. 2013.

[9] Principal Component Analysis, http://www.cse.unr.edu/ bebis/MathMethods/PCA/ lecture.pdf.

[10] Rade Kutil, Dominik Engel, Methods for the anisotropic wavelet packet transform, Applied and Computational Harmonic Analysis, Vol. 25, Issue 3, November 2008, pp. 295-314. 